\title{
Mutations in the noncoding genome contribute to autism
}

\author{
BY LAUREN SCHENKMAN
}

2 AUGUST 2021

Spontaneous mutations in 'noncoding' regions of the genome are linked to autism, according to a new study. The work provides strong support for the idea that autism's genetic roots lie not only in protein-coding genes but also in non-gene DNA.

"This is substantial evidence that these noncoding mutations can have a strong phenotypic impact," says Alex Nord, associate professor of psychiatry at the University of California, Davis, who was not involved in the new work.

Researchers have long focused on autism-related mutations in genes, which code for proteins and make up only 2 percent of the genome. The other 98 percent of the genome is 'noncoding': It doesn't contain instructions for proteins but instead influences when, where and how much genes are expressed.

Statistics-based studies have suggested that the genome's noncoding region contains genetic variants that contribute to autism, but the new work may be the first to identify three such mutations, says Tychele Turner, assistant professor of genetics at Washington University in St. Louis, who led the new work.

"I just thought it was cool that we can begin to get into the noncoding variation space," Turner says. "Maybe in three or four years, we'll have a pretty cool list [of additional noncoding variants]."

\section{Unexplored territory:}

Turner and her team searched a database of 544 noncoding segments of the genome shown to be active in the brains of mouse embryos. These so-called enhancer segments increase gene transcription. 


\section{Spectrum | Autism Research News}

https://www.spectrumnews.org

They then searched whole genome sequences from 2,671 families with one autistic child in the Simons Simplex Collection database to see which of these enhancers contained de novo variants. (The Simons Simplex Collection is funded by the Simons Foundation, Spectrum's parent organization.)

One enhancer in particular, known as hs737, showed significantly more de novo mutations in both a discovery cohort of 516 families and a replication cohort of 2,155 families. Notably, these de novo mutations in hs737 appeared in three people with autism with no other known autism-linked variants.

In experiments with neuronal cell lines, the three de novo mutations caused the enhancer to lower, not boost, gene expression, the researchers found. Existing maps of genome interactions in mouse and human brain cells showed that $\mathrm{h} 737$ regulates a gene called EBF3. The team shared preliminary results at the 2020 American Society of Human Genetics Conference and published them on 13 July in Human Genomics.

To the researchers' surprise, EBF3 is a well-studied gene. Mutations in EBF3 cause a condition called hypotonia, ataxia and delayed development syndrome (HADDS), characterized by low muscle tone and motor problems. Autistic people with mutations in hs737 also had motor problems or low muscle tone, the team found. That may be because - even though EBF3 is widely expressed through the body - hs737 is active in the brain only while an embryo is developing, Turner says. So mutations in the enhancer might lead to similar but less severe traits than mutations in the gene itself.

"This is really interesting," Turner says. "It's starting to tease apart ... why some people might have these big syndromes, but if you only affected a tissue-specific regulator of that gene, [the effect] might not be as severe, but it will still be important."

\section{Only the beginning:}

The statistical significance of hs737's link to autism is marginal, but the follow-up work done by Turner and her colleagues to establish the link "is to me very convincing," says Dan Arking, professor of genetic medicine at Johns Hopkins University School of Medicine, who was not involved in the work.

The work provides "a nice model" of how to link a noncoding mutation with a phenotype, he says. "They captured lots of pieces, put the puzzle together, and did a really nice job with it."

Even though only one enhancer rose to the level of nominal significance in this study, in time more enhancers may well be linked to autism, both the researchers and other scientists say.

It's as if "at first we were looking in a pond for fish and then we moved out to a giant lake," Nord 


\section{Spectrum | Autism Research News}

https://www.spectrumnews.org

says. That means that more samples are needed to establish significance, but "this is the first thing, not the only thing."

Turner's lab is working on developing a strain of mice that lacks the hs737 enhancer, in order to better understand how mutations in that stretch of noncoding DNA affect behavior.

Cite this article: https://doi.org/10.53053/VCFT1127 